$63^{\text {ème }}$ Congrès de la SFCO, 03004 (2015)

DOI:10.1051/sfco/20156303004

(C) Owned by the authors, published by EDP Sciences, 2015

POSTER

\title{
Techniques d'élévation du plancher sinusien par voie latérale sans particules de comblement : mise au point
}

\author{
Saad $\mathrm{R}^{* * * *}$, Keller $\mathrm{P}^{* * *}$, Lafon $\mathrm{A}^{* * * *}$, Boukari $\mathrm{A}^{* * * * *}$, Meyer $\mathrm{F}^{* * * * * * *}$, Talbi $\mathrm{M}^{* *}$, \\ Bornert $\mathrm{F}^{* * * * *}$ \\ * Faculté de Chirurgie-Dentaire de Strasbourg \\ ** Service de Chirurgie Maxillo-Faciale, Plastique et Stomatologie de l'hôpital de Mercy, Metz \\ *** Unité de Chirurgie buccale, Hôpitaux Universitaires de Strasbourg \\ **** Service d'Odontologie de l'hôpital Général de Dijon \\ ****** Unité d'Odontologie Conservatrice, Hôpitaux Universitaires de Strasbourg
}

La technique d'élévation sinusienne par voie latérale a été décrite dès les années 80 par Boyne et Tatum, et s'est très largement diffusée dans le monde depuis. De nombreux matériaux de comblement ont été testés afin de trouver le matériau idéal, initialement de l'os autogène. Puis, du fait d'un taux de résorption non prédictible associé au risque de morbidité du site donneur, des substituts osseux sont progressivement apparus sur le marché. Ils sont actuellement largement utilisés par les praticiens (Hallmanand et al. 2008). Plus récemment, des études portant sur des mélanges os autogène-substituts osseux combinés ou non à des facteurs de croissance ont été menées, avec un suivi à long terme satisfaisant et un taux de survie similaire au comblement par de l'os autogène (Voss et al. 2010). Le comblement du sinus avec particules de comblement constitue ainsi le gold standard. Cependant, il subsiste des inconvénients tels que la morbidité du site donneur ou encore le coût des biomatériaux.

La problématique de ce travail est la suivante : peut-on proposer des " greffes » sinusiennes par abord latéral sans utiliser des particules de comblement et espérer des taux de succès similaires aux techniques du gold standard?

Ce travail vise ainsi à faire le point et à comparer les résultats cliniques et histologiques disponibles dans la littérature afin d’apporter un éclairage sur ces techniques séduisantes.

Différentes techniques n'utilisant pas de particules de comblement sont décrites dans diverses études, par l'utilisation exclusive d'un caillot sanguin (Lundgren et al. 2004, Thor et al. 2007, Moon et al. 2011, Cricchio et al. 2011), de caillot et de membranes de PRF (Mazor et al. 2009, Simonpieri et al. 2011, Sohn et al. 2011), d'éponge de gélatine (Sohn et al. 2010), de mainteneur d'espace résorbable (Felice et al. 2009) ou encore de mainteneur d'espace en titane (Kaneko et al. 2012, Atef et al. 2013). L'utilisation d'une membrane de recouvrement, du repositionnement du volet osseux et la réalisation d'une implantation immédiate (de 3 à $5 \mathrm{~mm}$ minimum d'os nécessaire selon les auteurs) sont également discutés. Les résultats de ces techniques sont comparés entre eux ainsi qu'à ceux du gold standard notamment vis-à-vis du taux de succès, du risque de morbidité et des complications.

Les études cliniques, même si elles comportent certaines insuffisances sur le plan méthodologique, semblent indiquer que le comblement sinusien sans particules est une technique fiable avec des études

This is an Open Access article distributed under the terms of the Creative Commons Attribution License 4.0, which permits unrestricted use, distribution, and reproduction in any medium, provided the original work is properly cited. 
affichant 98 \% de taux de succès. L' « effet tente » lié à la mise en place concomitante des implants semble être le facteur déterminant pour le succès de cette technique.

Nom et adresse du conférencier

Ryan SAAD

Faculté de Chirurgie Dentaire de Strasbourg

8 rue Sainte-Elisabeth

67000 Strasbourg (France)

saad.ryan@hotmail.fr 